\title{
Organic Letters
}

Supplementary Information for

\section{Rapid Screening of a Receptor with Molecular Memory}

\author{
Authors:
}

\section{Judith M. Lavin, Ken D. Shimizu*}

Department of Chemistry and Biochemistry, University of South Carolina, Columbia, SC 29208 Fax: (803)7779521; Tel: (803)7776523

E-mail:shimizu@mail.chem.sc.edu 


\section{1) General Experimental:}

NMR spectra were recorded on Varian $300 \mathrm{MHz}, 400 \mathrm{MHz}$ and $500 \mathrm{MHz}$ spectrometers. Chemical shifts are reported in ppm $(\delta)$ referenced to TMS $(\delta 0.00 \mathrm{ppm})$ in ${ }^{1} \mathrm{H}$ NMR. All chemicals were purchased from commercial suppliers and used as received unless otherwise specified. Flash chromatography using silica (Sorbent Technologies, silica gel $60 \AA$ A, 200-400 mesh). Thin layer chromatography (TLC) was performed on precoated TLC plates (Merck precoated $0.25 \mathrm{~mm}$ silica gel $60 \mathrm{~F}_{254}$ plates). HPLC was performed using a Varian Prostar HPLC model 210 equipped with a Varian Prostar UV-Vis detector model 320, and a Rainin Dynamax $60 \AA$ silica column. UV detection was performed at $400 \mathrm{~nm}$.

\section{2) Synthesis}

Diol (2). A solution of 2-amino-4-tert-amylphenol (5.00 g, $0.0279 \mathrm{~mol})$ and 1,4,5,8naphthalenetetracarboxylic anhydride $(3.74 \mathrm{~g}, \quad 0.0139 \mathrm{~mol})$ in anhydrous dimethylformamide $(30 \mathrm{~mL})$ was heated at reflux at $150{ }^{\circ} \mathrm{C}$ for $12 \mathrm{~h}$. The reaction mixture was cooled to room temperature and the product precipitated out of acidic water (500 mL H $\mathrm{H}_{2} \mathrm{O}, 50 \mathrm{~mL} \mathrm{1N} \mathrm{HCl).} \mathrm{The} \mathrm{product} \mathrm{(2),} \mathrm{obtained} \mathrm{as} \mathrm{a} \mathrm{mixture} \mathrm{of} \mathrm{syn} \mathrm{and} \mathrm{anti}$ isomers, was filtered and dried under vacuum overnight. A brown powder was obtained in a $78 \%(9.00 \mathrm{~g})$ yield and used without further purification. ${ }^{1} \mathrm{H}$ NMR $\left(\mathrm{d}_{6}\right.$-acetone, 300 MHz) $\delta$ ppm: $8.365(\mathrm{~s}, 4 \mathrm{H}), 7.361(\mathrm{dd}, J=2.4 \mathrm{~Hz}, J=8.4 \mathrm{~Hz}, 2 \mathrm{H}), 7.312(\mathrm{~d}, J=2.4 \mathrm{~Hz}$, 2H), $7.033(\mathrm{~d}, J=8.4 \mathrm{~Hz}, 2 \mathrm{H}), 1.649$ (q, $J=7.2 \mathrm{~Hz}, 4 \mathrm{H}), 1.277(\mathrm{~s}, 12 \mathrm{H}), 0.7438$ (t, $J=$ 7.4 Hz, 6H). ${ }^{13} \mathrm{C}$ NMR (d $\mathrm{d}_{6}$-acetone, $\left.125 \mathrm{MHz}\right) \delta \mathrm{ppm}: 162.88,151.28,140.89,130.75$, 
127.91, 127.67, 127.53, 116.35, 37.30, 36.98, 8.89. HRMS (ESI): calculated for $\mathrm{C}_{36} \mathrm{H}_{34} \mathrm{~N}_{2} \mathrm{O}_{6}\left(\mathrm{M}^{+}\right)$, 591.2495; found, 591.2497.

Di-t-butylester (3). A solution of diol (2) $(0.5 \mathrm{~g}, 0.0012 \mathrm{~mol})$ and $t$-butylbromoacetate $(0.38 \mathrm{~mL}, 0.0026 \mathrm{~mol})$ in anhydrous dimethylformamide $(10 \mathrm{~mL})$ was stirred under nitrogen for 10 min before addition of potassium carbonate $(1.67 \mathrm{~g}, 0.012 \mathrm{~mol})$. The reaction mixture was stirred at room temperature under nitrogen for $12 \mathrm{~h}$. The product was precipitated out of acidic water $\left(200 \mathrm{~mL} \mathrm{H}_{2} \mathrm{O}, 50 \mathrm{~mL} 1 \mathrm{~N} \mathrm{HCl}\right)$. The product (3) was filtered and dried under vacuum overnight. A brown flaky solid was obtained in a $87 \%(0.68 \mathrm{~g})$ yield. ${ }^{1} \mathrm{H}$ NMR $\left(\mathrm{CDCl}_{3}, 300 \mathrm{MHz}\right) \delta \mathrm{ppm}: 8.812(\mathrm{~s}, 4 \mathrm{H}), 7.411(\mathrm{dd}, J=2.1$ $\mathrm{Hz}, J=8.4 \mathrm{~Hz}, 2 \mathrm{H}), 7.249(\mathrm{~d}, J=2.4 \mathrm{~Hz}, 2 \mathrm{H}), 6.928(\mathrm{~d}, J=8.7 \mathrm{~Hz}, 2 \mathrm{H}), 4.461(\mathrm{~s}, 4 \mathrm{H})$, $1.689(\mathrm{q}, J=7.5 \mathrm{~Hz}, 4 \mathrm{H}), 1.479(\mathrm{~s}, 12 \mathrm{H}), 1.325(\mathrm{~s}, 18 \mathrm{H}), 0.757(\mathrm{t}, J=7.5 \mathrm{~Hz}, 6 \mathrm{H}) .{ }^{13} \mathrm{C}$ NMR (d $\mathrm{d}_{6}$-acetone, $\left.125 \mathrm{MHz}\right) \delta \mathrm{ppm}: 167.44,152.06,142.94,130.92,128.35,127.53$, 113.51, 81.33, 66.72, 37.51, 36.95, 8.89. HRMS (ESI): calculated for $\mathrm{C}_{48} \mathrm{H}_{54} \mathrm{~N}_{2} \mathrm{O}_{10}\left(\mathrm{M}^{+}\right)$, 819.3857; found, 819.3833.

Diacid (1). Diester (3) $(0.10 \mathrm{~g}, 0.156 \mathrm{mmol})$ was dissolved in methylene chloride (8 $\mathrm{mL})$. Trifluoroacetic acid (4 mL) was slowly added to the solution. The reaction was stirred at room temperature for $12 \mathrm{~h}$. Additional methylene chloride $(40 \mathrm{~mL})$ was added to the reaction mixture and the solution was washed with acidic water $\left(25 \mathrm{~mL} \mathrm{H}_{2} \mathrm{O}, 10\right.$ $\mathrm{mL} 1 \mathrm{~N} \mathrm{HCl}$ ) three times, dried over anhydrous magnesium sulfate, filtered and concentrated. The crude mixture was purified by flash chromatography on silica gel 
using methylene chloride/acetic acid (95:5) as the eluting solvent. A 57.2\% yield of the anti isomer and a $21.1 \%$ yield of syn isomer were obtained upon purification.

${ }^{1} \mathrm{H}$ NMR, Anti-1 (d $\mathrm{d}_{6}$-acetone, $\left.300 \mathrm{MHz}\right) \delta$ ppm: 8.784 (s, 4H), 7.478 (m, 4H), 7.159 (d, J $=9.3 \mathrm{~Hz}, 2 \mathrm{H}), 4.696(\mathrm{~s}, 4 \mathrm{H}), 1.694(\mathrm{q}, J=7.5 \mathrm{~Hz}, 4 \mathrm{H}), 1.328(\mathrm{~s}, 12 \mathrm{H}), 0.768(\mathrm{t}, J=7.2$

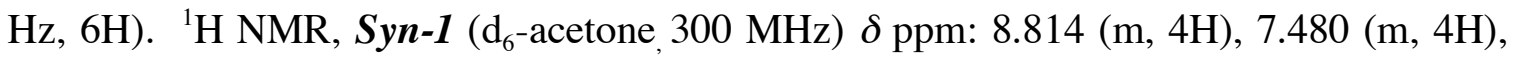
$7.155(\mathrm{~d}, J=9.3 \mathrm{~Hz}, 2 \mathrm{H}), 4.658(\mathrm{~s}, 4 \mathrm{H}), 1.688(\mathrm{q}, J=7.5 \mathrm{~Hz}, 4 \mathrm{H}), 1.320(\mathrm{~s}, 12 \mathrm{H}), 0.768$ $(\mathrm{t}, J=7.2 \mathrm{~Hz}, 6 \mathrm{H}) .{ }^{13} \mathrm{C}$ NMR Anti-1 (d $\mathrm{d}_{6}$-acetone, $\left.125 \mathrm{MHz}\right) \delta \mathrm{ppm}: 171.915,169.377$, $162.634,152.008,143.148,130.994,128.405,127.677,127.401,124.579,113.661$, 66.027, 37.536, 36.918, 19.927, 8.914. ${ }^{13} \mathrm{C}$ NMR Syn-1 (d -acetone, $\left.125 \mathrm{MHz}\right) \delta \mathrm{ppm}:$ $169.186,162.705,152.107,143.131,130.904,128.387,127.623,124.772,113.883$, 66.174, 37.494, 36.875, 8.806. Anti-1 HRMS (ESI): calculated for $\mathrm{C}_{40} \mathrm{H}_{38} \mathrm{~N}_{2} \mathrm{O}_{10}(\mathrm{M}+$ ), 707.2604; found, 707.2582. Syn-1 HRMS (ESI): calculated for $\mathrm{C}_{40} \mathrm{H}_{38} \mathrm{~N}_{2} \mathrm{O}_{10}(\mathrm{M}+$ ), 707.2604; found, 707.2592.

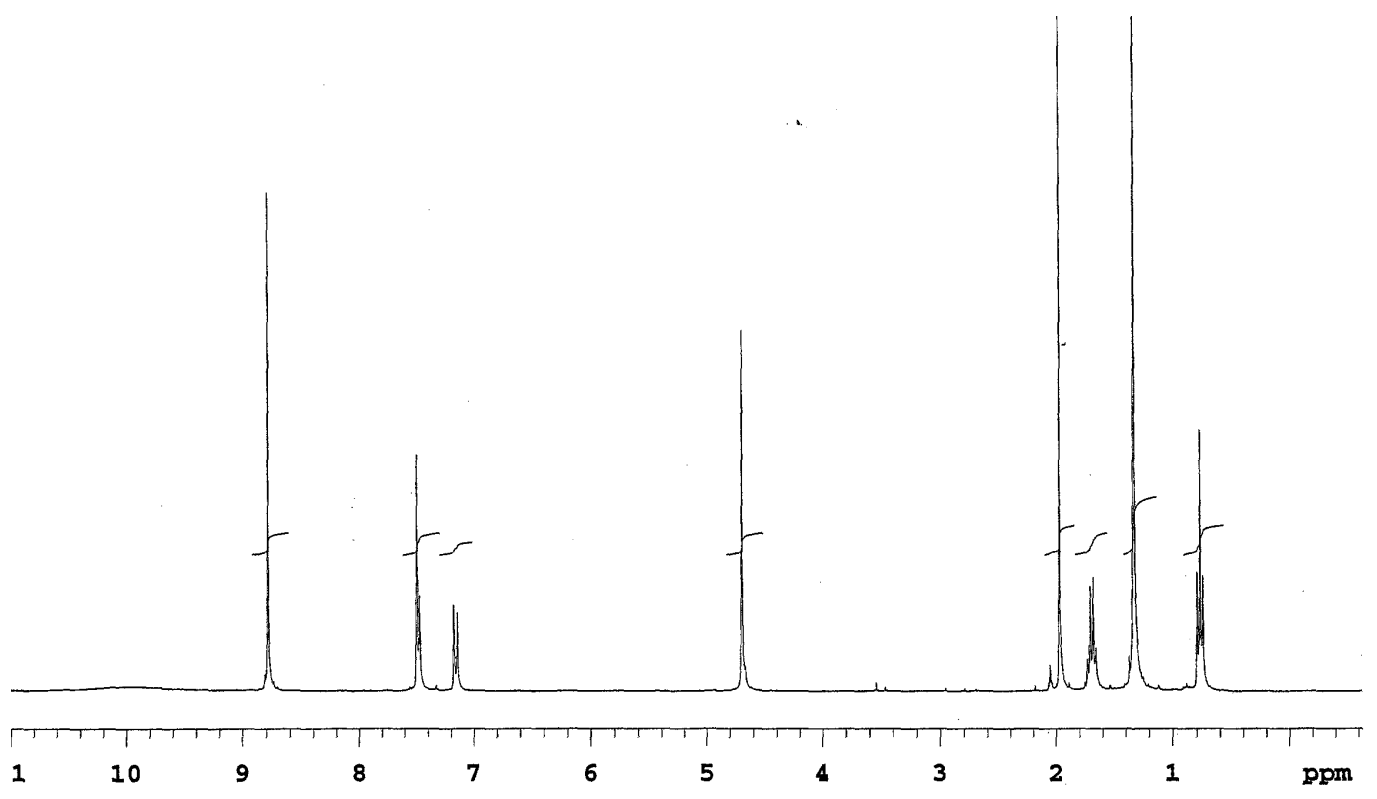

Figure S1: ${ }^{1} \mathrm{H}$ NMR spectra of $\boldsymbol{A n t i - 1}$ in $d_{6}$-acteone. 
Shimizu et al.

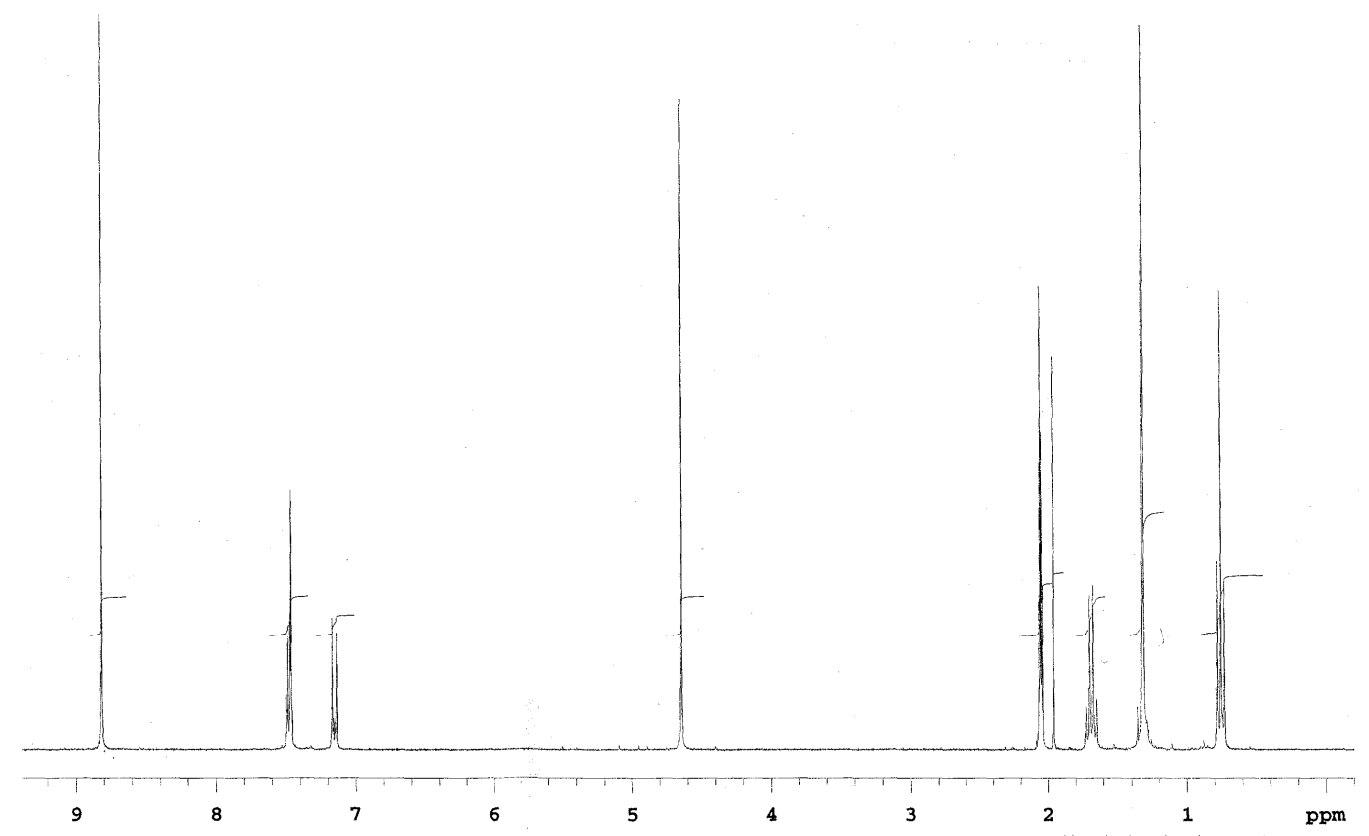

Figure S2: ${ }^{1} \mathrm{H}$ NMR spectra of $\mathbf{S y n - 1}$ in $d_{6}$-acteone.

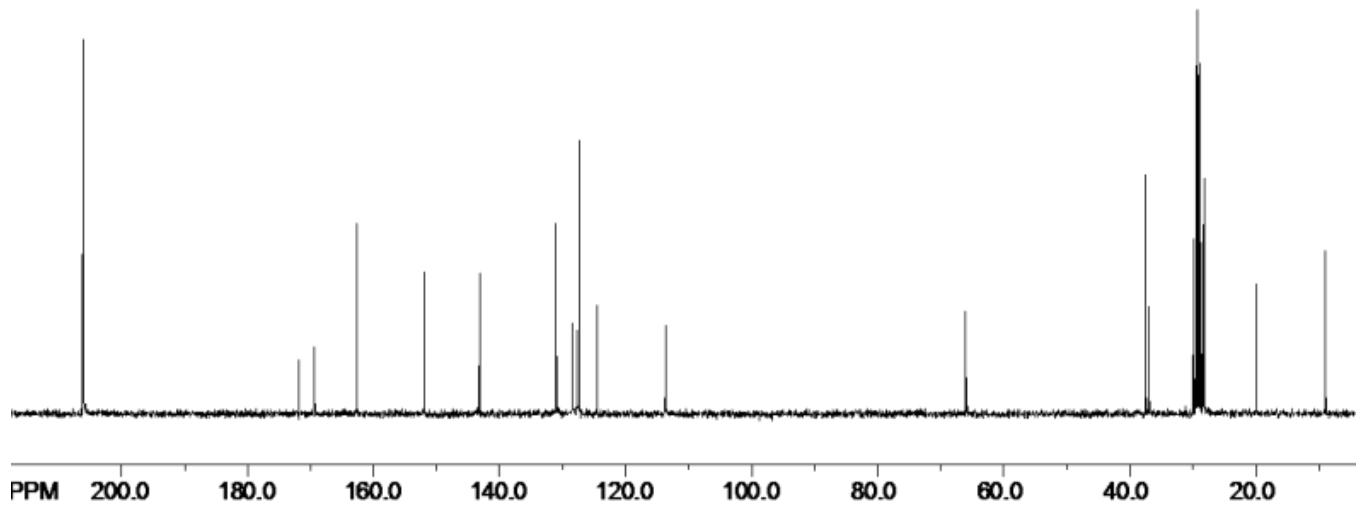

Figure S3: ${ }^{13} \mathrm{C}$ NMR anti-1 ( $\mathrm{d}_{6}$-acetone) 


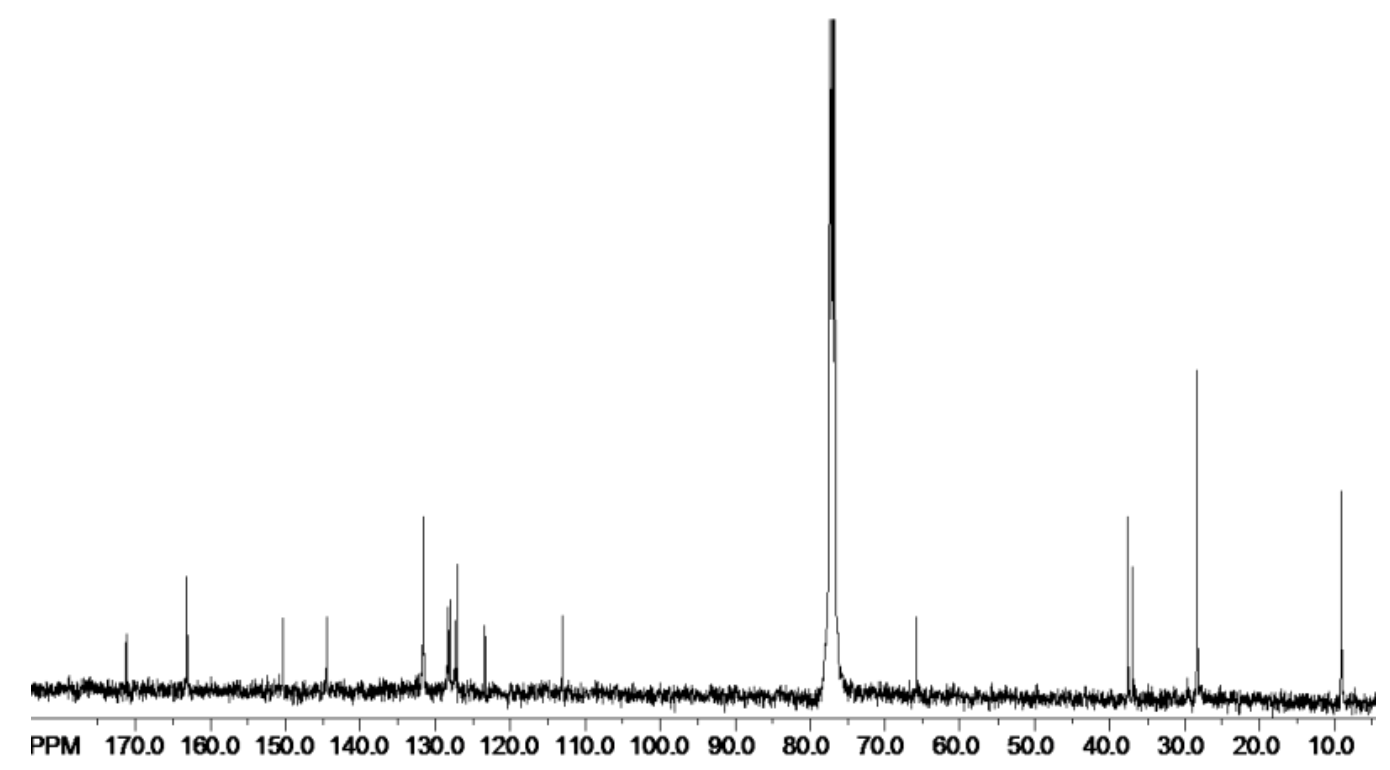

Figure S4: ${ }^{13} \mathrm{C}$ NMR syn-1 $\left(\mathrm{CDCl}_{3}\right)$

\section{3) Binding Study:}

An equilibrium mixture of diacid $\left(9.0 \mathrm{mg}, 1.2 \times 10^{-5} \mathrm{~mol}\right)$ and the nucleoside guest molecules (4-8) $\left(1.2 \times 10^{-5} \mathrm{~mol}\right)$ were dissolved in tetrachloroethane (TCE) and heated in an oil bath for 3 hours at $70{ }^{\circ} \mathrm{C}$. A $5 \mathrm{mM}$ solution of diacid $\left(9.0 \mathrm{mg}, 1.2 \times 10^{-5} \mathrm{~mol}\right)$ dissolved in TCE was heated in the presence of a guest molecule (8-15) $\left(1.2 \times 10^{-5} \mathrm{~mol}\right)$ at $70{ }^{\circ} \mathrm{C} 12$ hours. The syn/anti ratio was measured by on a Rainin Dynamax $60 \AA$ silica column with $12.5 \% \mathrm{CH}_{3} \mathrm{CO}_{2} \mathrm{H} / \mathrm{CHCl}_{3}$ as mobile phase with a flow rate of $1 \mathrm{~mL} / \mathrm{min}$.

\section{4) Rotational Barrier $\left(\Delta G^{\dagger+}\right)$ Heating Study of Syn Isomer.}

Syn diacid (12 mg) was dissolved in $\mathrm{d}_{6}$-TCE $(600 \mu \mathrm{L})$. FID's were recorded every 5 min for the first $20 \mathrm{~min}$ and then every $10 \mathrm{~min}$ for the next $23 \mathrm{~h}$. The study was carried out on a $500 \mathrm{mHz}$ Varian NMR temperature controlled at $65{ }^{\circ} \mathrm{C}$. The ratio of the two 
isomers was determined by standard deconvolution of the methylene protons at $4.66 \mathrm{ppm}$. The $\ln \left[\left(R-R_{e}\right) /(R+1)\right.$ of was plotted versus time $(s)$, where $R_{e}$ is the ratio of isomers at equilibrium and $\mathrm{R}$ the ratio of anti/syn at time $\mathrm{t}$ seconds. The slope of this plot corresponds to the observed rate $k_{\mathrm{obs}}$, where $k_{\mathrm{obs}}$ is equal to $4 k$. $k$ is the rate of rotation of a single rotor for a reversible reaction. The 4 accounts for the reversibility of the system (the forward and the reverse rate constants) and the two rotors. Then using the Eyring equation the rotational barrier can be determined. The energy of rotation was calculated to be $26.51 \mathrm{kcal} / \mathrm{mol}$ at $65{ }^{\circ} \mathrm{C}$. The half-life, $\mathrm{t}_{1 / 2}$, calculated using $k_{\text {obs }}$, equals $(\ln 2) /(4$ $\left.\mathrm{k}_{\mathrm{obs}}\right)$.

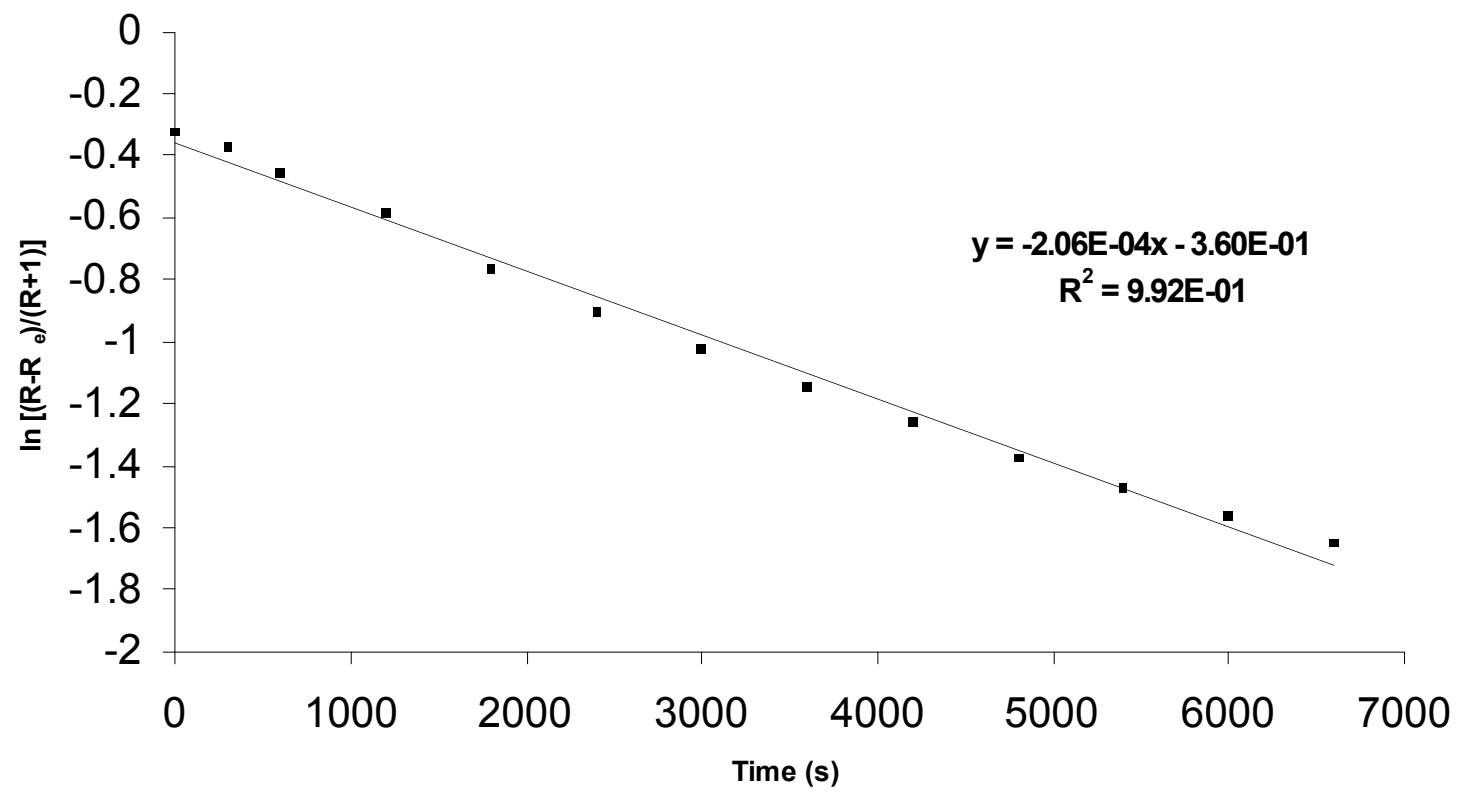

Figure S5: Rotational barrier determination via isomeric equilibration.

\section{5) Titration Study:}

The nucleotide base $(2 \mathrm{mM}, 600 \mu \mathrm{L})$ was titrated with a series of volumes of the isomerically pure diacid $(5 \mathrm{mM})$ in $\mathrm{CD}_{3} \mathrm{CN} .{ }^{1} \mathrm{H}$ NMR spectra were taken after each addition. The peak followed for the determination of the binding constant differed for 
each nucleoside base due to problems with overlapping and disappearing peaks. The host induced chemical shift data was then fit to a 1:1 binding isotherm. ${ }^{1}$ The solver function as implemented in Excel was used to identify which 1:1 binding isotherm yielded the best fit to the experimental data. The association constant $\left(K_{a}\right)$ of the 1:1 binding isotherm with the best fit was the reported $K_{a}$.

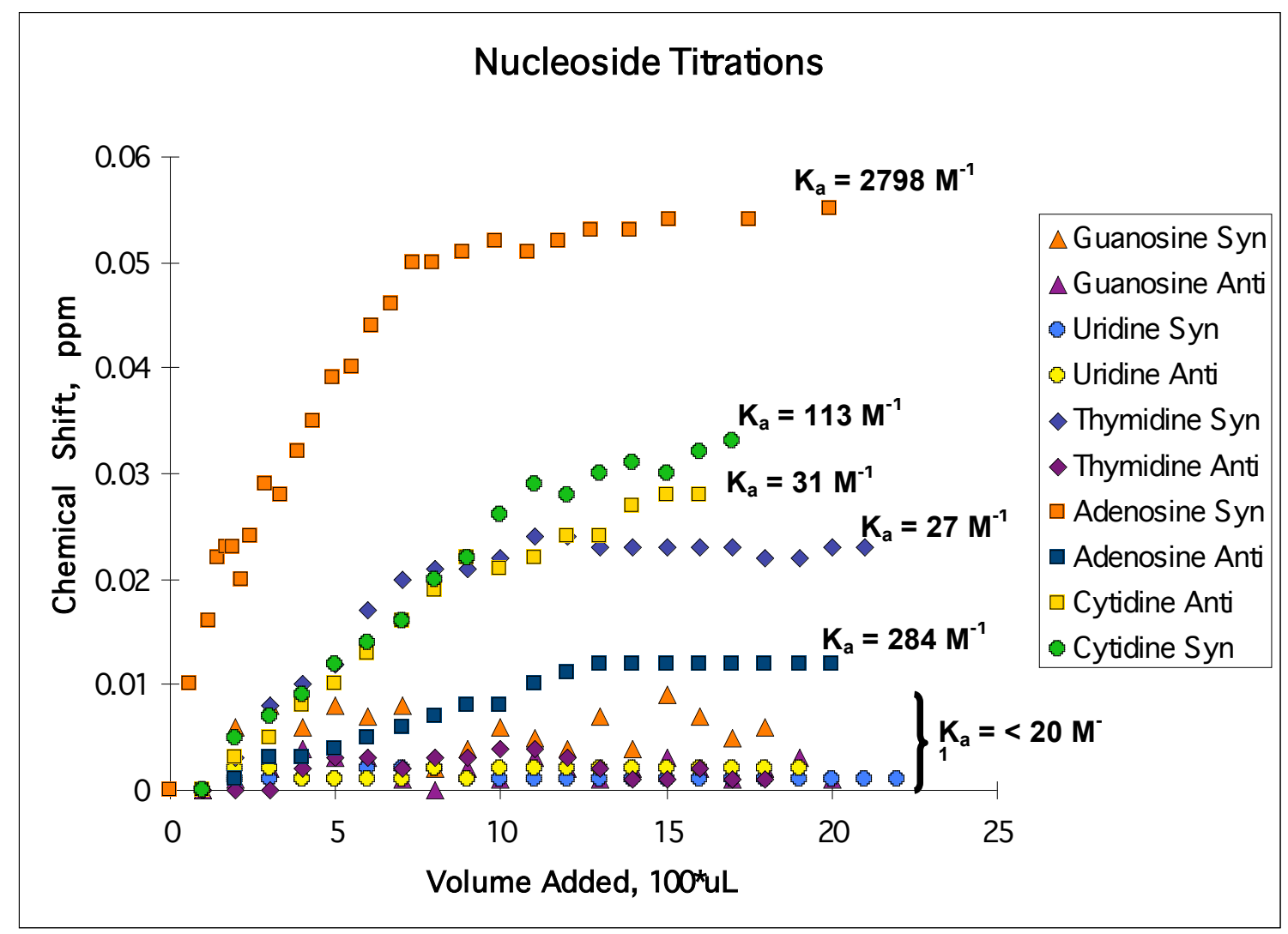

Figure S6: Titration plot of nucleoside bases with syn and anti-1.

\section{6) Crystallographic Data}

Crystals were obtained of the more quickly eluting isomer from $\mathrm{CH}_{2} \mathrm{Cl}_{2} / \mathrm{MeOH}$. The structure is the anti-isomer with the carboxylic acid moieties lying both above and 
below the naphthalene plane. The x-ray structure also gave confirmation of the expected rigid structure. The phenyl and naphthalenediimide surfaces are twisted out of plane with a dihedral angle of $79.9^{\circ}$. The carboxylic acids of anti-1 clearly cannot bind a single guest molecule. The carboxylic acids of anti-1 are directed inward toward the naphthalenediimide spacer, suggesting that the two carboxylic acids would be capable of binding a guest molecule in the corresponding syn-isomer.

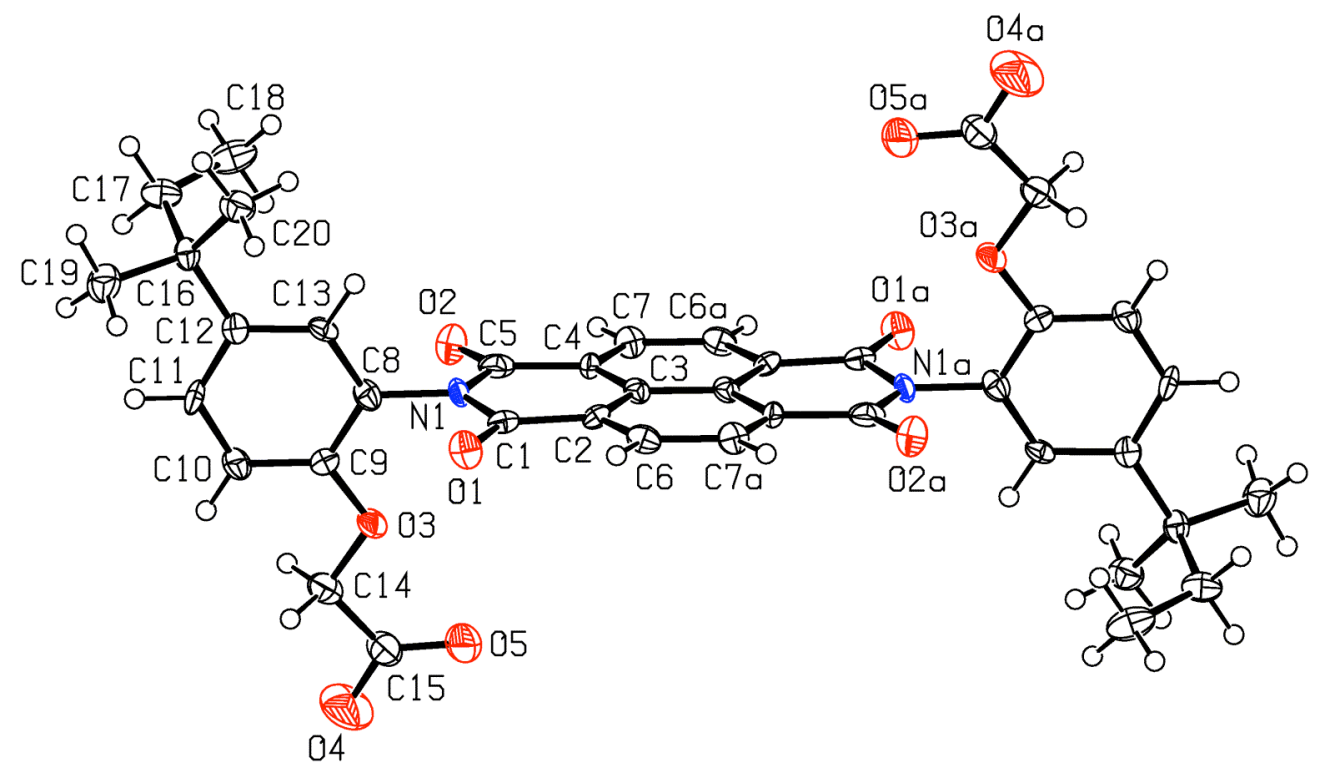

Figure S7: Molecular structure of anti-1 with phenyl and naphthalenediimide surfaces twisted out of plane with a dihedral angle of $79.9^{\circ}$. 
Table 1. Crystal data and structure refinement for sqz.

Identification code

Empirical formula

Formula weight

Temperature

Wavelength

Crystal system

Space group

Unit cell dimensions

Volume

Z

Density (calculated)

Absorption coefficient

$\mathrm{F}(000)$

Crystal size

Theta range for data collection

Index ranges

Reflections collected

Independent reflections

Completeness to theta $=20.18^{\circ}$

Absorption correction

Refinement method

Data / restraints / parameters

Goodness-of-fit on $\mathrm{F}^{2}$

Final $R$ indices $[\mathrm{I}>2 \operatorname{sigma}(\mathrm{I})]$

$\mathrm{R}$ indices (all data)

Largest diff. peak and hole $\mathrm{sqz}$

C40 H38 N2 O10

706.72

100(1) K

$0.71073 \AA$

Trigonal

R-3

$$
\begin{array}{ll}
\mathrm{a}=31.7102(11) \AA & \alpha=90^{\circ} . \\
\mathrm{b}=31.7102(11) \AA & \beta=90^{\circ} . \\
\mathrm{c}=9.7910(8) \AA & \gamma=120^{\circ} .
\end{array}
$$

8526.2(8) $\AA^{3}$

9

$1.239 \mathrm{Mg} / \mathrm{m}^{3}$

$0.090 \mathrm{~mm}^{-1}$

3348

$0.14 \times 0.08 \times 0.06 \mathrm{~mm}^{3}$

1.28 to $20.18^{\circ}$.

$-30<=\mathrm{h}<=30,-30<=\mathrm{k}<=30,-9<=1<=7$

9413

$1813[\mathrm{R}(\mathrm{int})=0.1088]$

$100.0 \%$

None

Full-matrix least-squares on $\mathrm{F}^{2}$

1813 / 0 / 256

1.009

$\mathrm{R} 1=0.0522, \mathrm{wR} 2=0.0840$

$\mathrm{R} 1=0.0931, \mathrm{wR} 2=0.0933$

0.194 and -0.156 e. $\AA^{-3}$ 
Table 2. Atomic coordinates $\left(\mathrm{x} 10^{4}\right)$ and equivalent isotropic displacement parameters $\left(\AA^{2} \times 10^{3}\right)$

for sqz. $U(\mathrm{eq})$ is defined as one third of the trace of the orthogonalized $U^{\mathrm{ij}}$ tensor.

\begin{tabular}{|c|c|c|c|c|}
\hline & $\mathrm{x}$ & $\mathrm{y}$ & $\mathrm{z}$ & $\mathrm{U}(\mathrm{eq})$ \\
\hline $\mathrm{C}(1)$ & $652(2)$ & $2878(2)$ & $4799(5)$ & $19(1)$ \\
\hline $\mathrm{C}(2)$ & $1044(2)$ & $3203(2)$ & $3866(5)$ & $16(1)$ \\
\hline$C(3)$ & $1483(2)$ & $3184(2)$ & $3806(5)$ & $18(1)$ \\
\hline$C(4)$ & $1563(2)$ & $2877(2)$ & $4667(5)$ & $19(1)$ \\
\hline$C(5)$ & $1185(2)$ & $2563(2)$ & $5670(6)$ & $26(1)$ \\
\hline$C(6)$ & $982(2)$ & $3516(2)$ & $3027(5)$ & $23(1)$ \\
\hline$C(7)$ & $1349(2)$ & $3814(2)$ & $2080(5)$ & $23(1)$ \\
\hline $\mathrm{C}(8)$ & $375(2)$ & $2281(2)$ & $6642(5)$ & $22(1)$ \\
\hline $\mathrm{C}(9)$ & $71(2)$ & $1801(2)$ & $6328(5)$ & $22(1)$ \\
\hline$C(10)$ & $-298(2)$ & $1511(2)$ & $7241(6)$ & $26(1)$ \\
\hline $\mathrm{C}(11)$ & $-338(2)$ & $1709(2)$ & $8461(5)$ & $27(1)$ \\
\hline$C(12)$ & $-37(2)$ & 2193(2) & $8816(5)$ & $24(1)$ \\
\hline$C(13)$ & $321(2)$ & $2474(2)$ & $7870(5)$ & $18(1)$ \\
\hline$C(14)$ & $-151(2)$ & $1130(2)$ & $4866(6)$ & $31(2)$ \\
\hline$C(15)$ & $-14(2)$ & $1000(2)$ & $3542(6)$ & $34(2)$ \\
\hline$C(16)$ & $-70(2)$ & $2388(2)$ & $10204(5)$ & $21(1)$ \\
\hline$C(17)$ & $212(2)$ & $2270(2)$ & $11263(6)$ & $30(2)$ \\
\hline$C(18)$ & $749(2)$ & $2465(2)$ & 10987(6) & $42(2)$ \\
\hline$C(19)$ & $-603(2)$ & $2144(2)$ & $10710(6)$ & $35(2)$ \\
\hline$C(20)$ & $115(2)$ & $2939(2)$ & $10173(6)$ & $30(2)$ \\
\hline $\mathrm{N}(1)$ & $753(1)$ & $2593(1)$ & $5691(4)$ & $18(1)$ \\
\hline $\mathrm{O}(1)$ & $257(1)$ & $2851(1)$ & $4818(4)$ & $27(1)$ \\
\hline $\mathrm{O}(2)$ & $1226(1)$ & $2292(1)$ & $6465(4)$ & $31(1)$ \\
\hline $\mathrm{O}(3)$ & 149(1) & $1638(1)$ & $5111(4)$ & $28(1)$ \\
\hline $\mathrm{O}(4)$ & $-251(1)$ & $540(1)$ & $3285(5)$ & $70(1)$ \\
\hline $\mathrm{O}(5)$ & $298(1)$ & $1315(1)$ & $2754(4)$ & $46(1)$ \\
\hline
\end{tabular}


Table 3. Bond lengths $[\AA]$ and angles $\left[^{\circ}\right]$ for sqz.

\begin{tabular}{ll}
\hline $\mathrm{C}(1)-\mathrm{O}(1)$ & $1.215(5)$ \\
$\mathrm{C}(1)-\mathrm{N}(1)$ & $1.401(6)$ \\
$\mathrm{C}(1)-\mathrm{C}(2)$ & $1.469(6)$ \\
$\mathrm{C}(2)-\mathrm{C}(6)$ & $1.375(6)$ \\
$\mathrm{C}(2)-\mathrm{C}(3)$ & $1.424(6)$ \\
$\mathrm{C}(3)-\mathrm{C}(4)$ & $1.406(6)$ \\
$\mathrm{C}(3)-\mathrm{C}(3) \# 1$ & $1.417(9)$ \\
$\mathrm{C}(4)-\mathrm{C}(7) \# 1$ & $1.379(6)$ \\
$\mathrm{C}(4)-\mathrm{C}(5)$ & $1.483(7)$ \\
$\mathrm{C}(5)-\mathrm{O}(2)$ & $1.214(5)$ \\
$\mathrm{C}(5)-\mathrm{N}(1)$ & $1.420(6)$ \\
$\mathrm{C}(6)-\mathrm{C}(7)$ & $1.417(6)$ \\
$\mathrm{C}(6)-\mathrm{H}(6)$ & 0.9500 \\
$\mathrm{C}(7)-\mathrm{C}(4) \# 1$ & $1.379(6)$ \\
$\mathrm{C}(7)-\mathrm{H}(7)$ & 0.9500 \\
$\mathrm{C}(8)-\mathrm{C}(9)$ & $1.371(6)$ \\
$\mathrm{C}(8)-\mathrm{C}(13)$ & $1.398(7)$ \\
$\mathrm{C}(8)-\mathrm{N}(1)$ & $1.447(6)$ \\
$\mathrm{C}(9)-\mathrm{O}(3)$ & $1.370(6)$ \\
$\mathrm{C}(9)-\mathrm{C}(10)$ & $1.390(6)$ \\
$\mathrm{C}(10)-\mathrm{C}(11)$ & $1.385(7)$ \\
$\mathrm{C}(10)-\mathrm{H}(10)$ & 0.9500 \\
$\mathrm{C}(11)-\mathrm{C}(12)$ & $1.386(6)$ \\
$\mathrm{C}(11)-\mathrm{H}(11)$ & 0.9500 \\
$\mathrm{C}(12)-\mathrm{C}(13)$ & $1.389(6)$ \\
$\mathrm{C}(12)-\mathrm{C}(16)$ & $1.518(7)$ \\
$\mathrm{C}(13)-\mathrm{H}(13)$ & 0.9500 \\
$\mathrm{C}(14)-\mathrm{O}(3)$ & $1.423(5)$ \\
$\mathrm{C}(14)-\mathrm{C}(15)$ & $1.488(7)$ \\
$\mathrm{C}(14)-\mathrm{H}(14 \mathrm{~A})$ & 0.9900 \\
$\mathrm{C}(14)-\mathrm{H}(14 \mathrm{~B})$ & 0.9900 \\
$\mathrm{C}(15)-\mathrm{O}(5)$ & $1.259(6)$ \\
$\mathrm{C}(15)-\mathrm{O}(4)$ & $1.288(6)$ \\
$\mathrm{C}(16)-\mathrm{C}(17)$ & $1.534(6)$ \\
&
\end{tabular}




$\begin{array}{lc}\mathrm{C}(16)-\mathrm{C}(20) & 1.540(6) \\ \mathrm{C}(16)-\mathrm{C}(19) & 1.548(6) \\ \mathrm{C}(17)-\mathrm{C}(18) & 1.516(6) \\ \mathrm{C}(17)-\mathrm{H}(17 \mathrm{~A}) & 0.9900 \\ \mathrm{C}(17)-\mathrm{H}(17 \mathrm{~B}) & 0.9900 \\ \mathrm{C}(18)-\mathrm{H}(18 \mathrm{~A}) & 0.9800 \\ \mathrm{C}(18)-\mathrm{H}(18 \mathrm{~B}) & 0.9800 \\ \mathrm{C}(18)-\mathrm{H}(18 \mathrm{C}) & 0.9800 \\ \mathrm{C}(19)-\mathrm{H}(19 \mathrm{~A}) & 0.9800 \\ \mathrm{C}(19)-\mathrm{H}(19 \mathrm{~B}) & 0.9800 \\ \mathrm{C}(19)-\mathrm{H}(19 \mathrm{C}) & 0.9800 \\ \mathrm{C}(20)-\mathrm{H}(20 \mathrm{~A}) & 0.9800 \\ \mathrm{C}(20)-\mathrm{H}(20 \mathrm{~B}) & 0.9800 \\ \mathrm{C}(20)-\mathrm{H}(20 \mathrm{C}) & 0.9800 \\ & \\ \mathrm{O}(1)-\mathrm{C}(1)-\mathrm{N}(1) & 120.2(4) \\ \mathrm{O}(1)-\mathrm{C}(1)-\mathrm{C}(2) & 122.7(5) \\ \mathrm{N}(1)-\mathrm{C}(1)-\mathrm{C}(2) & 117.1(4) \\ \mathrm{C}(6)-\mathrm{C}(2)-\mathrm{C}(3) & 120.0(5) \\ \mathrm{C}(6)-\mathrm{C}(2)-\mathrm{C}(1) & 120.2(4) \\ \mathrm{C}(3)-\mathrm{C}(2)-\mathrm{C}(1) & 119.8(4) \\ \mathrm{C}(4)-\mathrm{C}(3)-\mathrm{C}(3) \# 1 & 118.7(5) \\ \mathrm{C}(4)-\mathrm{C}(3)-\mathrm{C}(2) & 121.6(4) \\ \mathrm{C}(3) \# 1-\mathrm{C}(3)-\mathrm{C}(2) & 119.7(6) \\ \mathrm{C}(7) \# 1-\mathrm{C}(4)-\mathrm{C}(3) & 121.4(5) \\ \mathrm{C}(7) \# 1-\mathrm{C}(4)-\mathrm{C}(5) & 118.8(5) \\ \mathrm{C}(3)-\mathrm{C}(4)-\mathrm{C}(5) & 119.7(4) \\ \mathrm{O}(2)-\mathrm{C}(5)-\mathrm{N}(1) & 119.1(5) \\ \mathrm{O}(2)-\mathrm{C}(5)-\mathrm{C}(4) & 124.2(5) \\ \mathrm{N}(1)-\mathrm{C}(5)-\mathrm{C}(4) & 116.7(5) \\ \mathrm{C}(2)-\mathrm{C}(6)-\mathrm{C}(7) & 120.5(5) \\ \mathrm{C}(2)-\mathrm{C}(6)-\mathrm{H}(6) & 119.8 \\ \mathrm{C}(7)-\mathrm{C}(6)-\mathrm{H}(6) & 119.8 \\ \mathrm{C}(4) \# 1-\mathrm{C}(7)-\mathrm{C}(6) & 119.7(5) \\ \mathrm{C}(4) \# 1-\mathrm{C}(7)-\mathrm{H}(7) & 120.2 \\ \mathrm{C}(6)-\mathrm{C}(7)-\mathrm{H}(7) & 120.2 \\ & \end{array}$




$\begin{array}{ll}\mathrm{C}(9)-\mathrm{C}(8)-\mathrm{C}(13) & 120.4(5) \\ \mathrm{C}(9)-\mathrm{C}(8)-\mathrm{N}(1) & 119.9(5) \\ \mathrm{C}(13)-\mathrm{C}(8)-\mathrm{N}(1) & 119.7(4) \\ \mathrm{O}(3)-\mathrm{C}(9)-\mathrm{C}(8) & 117.0(5) \\ \mathrm{O}(3)-\mathrm{C}(9)-\mathrm{C}(10) & 124.2(5) \\ \mathrm{C}(8)-\mathrm{C}(9)-\mathrm{C}(10) & 118.8(5) \\ \mathrm{C}(11)-\mathrm{C}(10)-\mathrm{C}(9) & 119.6(5) \\ \mathrm{C}(11)-\mathrm{C}(10)-\mathrm{H}(10) & 120.2 \\ \mathrm{C}(9)-\mathrm{C}(10)-\mathrm{H}(10) & 120.2 \\ \mathrm{C}(10)-\mathrm{C}(11)-\mathrm{C}(12) & 123.2(5) \\ \mathrm{C}(10)-\mathrm{C}(11)-\mathrm{H}(11) & 118.4 \\ \mathrm{C}(12)-\mathrm{C}(11)-\mathrm{H}(11) & 118.4 \\ \mathrm{C}(11)-\mathrm{C}(12)-\mathrm{C}(13) & 115.7(5) \\ \mathrm{C}(11)-\mathrm{C}(12)-\mathrm{C}(16) & 121.7(5) \\ \mathrm{C}(13)-\mathrm{C}(12)-\mathrm{C}(16) & 122.4(4) \\ \mathrm{C}(12)-\mathrm{C}(13)-\mathrm{C}(8) & 122.2(5) \\ \mathrm{C}(12)-\mathrm{C}(13)-\mathrm{H}(13) & 118.9 \\ \mathrm{C}(8)-\mathrm{C}(13)-\mathrm{H}(13) & 118.9 \\ \mathrm{O}(3)-\mathrm{C}(14)-\mathrm{C}(15) & 109.8(4) \\ \mathrm{O}(3)-\mathrm{C}(14)-\mathrm{H}(14 \mathrm{~A}) & 109.7 \\ \mathrm{C}(15)-\mathrm{C}(14)-\mathrm{H}(14 \mathrm{~A}) & 109.7 \\ \mathrm{O}(3)-\mathrm{C}(14)-\mathrm{H}(14 \mathrm{~B}) & 109.7 \\ \mathrm{C}(15)-\mathrm{C}(14)-\mathrm{H}(14 \mathrm{~B}) & 109.7 \\ \mathrm{H}(14 \mathrm{~A})-\mathrm{C}(14)-\mathrm{H}(14 \mathrm{~B}) & 108.2 \\ \mathrm{O}(5)-\mathrm{C}(15)-\mathrm{O}(4) & 124.2(6) \\ \mathrm{O}(5)-\mathrm{C}(15)-\mathrm{C}(14) & 122.4(5) \\ \mathrm{O}(4)-\mathrm{C}(15)-\mathrm{C}(14) & 113.4(5) \\ \mathrm{C}(12)-\mathrm{C}(16)-\mathrm{C}(17) & 109.7(4) \\ \mathrm{C}(12)-\mathrm{C}(16)-\mathrm{C}(20) & 112.3(4) \\ \mathrm{C}(17)-\mathrm{C}(16)-\mathrm{C}(20) & 110.6(4) \\ \mathrm{C}(12)-\mathrm{C}(16)-\mathrm{C}(19) & 111.3(4) \\ \mathrm{C}(17)-\mathrm{C}(16)-\mathrm{C}(19) & 106.6(4) \\ \mathrm{C}(20)-\mathrm{C}(16)-\mathrm{C}(19) & 106.0(4) \\ \mathrm{C}(18)-\mathrm{C}(17)-\mathrm{C}(16) & 116.9(4) \\ \mathrm{C}(18)-\mathrm{C}(17)-\mathrm{H}(17 \mathrm{~A}) & 108.1 \\ \mathrm{C}(16)-\mathrm{C}(17)-\mathrm{H}(17 \mathrm{~A}) & 108.1 \\ & \end{array}$




\begin{tabular}{ll}
$\mathrm{C}(18)-\mathrm{C}(17)-\mathrm{H}(17 \mathrm{~B})$ & 108.1 \\
$\mathrm{C}(16)-\mathrm{C}(17)-\mathrm{H}(17 \mathrm{~B})$ & 108.1 \\
$\mathrm{H}(17 \mathrm{~A})-\mathrm{C}(17)-\mathrm{H}(17 \mathrm{~B})$ & 107.3 \\
$\mathrm{C}(17)-\mathrm{C}(18)-\mathrm{H}(18 \mathrm{~A})$ & 109.5 \\
$\mathrm{C}(17)-\mathrm{C}(18)-\mathrm{H}(18 \mathrm{~B})$ & 109.5 \\
$\mathrm{H}(18 \mathrm{~A})-\mathrm{C}(18)-\mathrm{H}(18 \mathrm{~B})$ & 109.5 \\
$\mathrm{C}(17)-\mathrm{C}(18)-\mathrm{H}(18 \mathrm{C})$ & 109.5 \\
$\mathrm{H}(18 \mathrm{~A})-\mathrm{C}(18)-\mathrm{H}(18 \mathrm{C})$ & 109.5 \\
$\mathrm{H}(18 \mathrm{~B})-\mathrm{C}(18)-\mathrm{H}(18 \mathrm{C})$ & 109.5 \\
$\mathrm{C}(16)-\mathrm{C}(19)-\mathrm{H}(19 \mathrm{~A})$ & 109.5 \\
$\mathrm{C}(16)-\mathrm{C}(19)-\mathrm{H}(19 \mathrm{~B})$ & 109.5 \\
$\mathrm{H}(19 \mathrm{~A})-\mathrm{C}(19)-\mathrm{H}(19 \mathrm{~B})$ & 109.5 \\
$\mathrm{C}(16)-\mathrm{C}(19)-\mathrm{H}(19 \mathrm{C})$ & 109.5 \\
$\mathrm{H}(19 \mathrm{~A})-\mathrm{C}(19)-\mathrm{H}(19 \mathrm{C})$ & 109.5 \\
$\mathrm{H}(19 \mathrm{~B})-\mathrm{C}(19)-\mathrm{H}(19 \mathrm{C})$ & 109.5 \\
$\mathrm{C}(16)-\mathrm{C}(20)-\mathrm{H}(20 \mathrm{~A})$ & 109.5 \\
$\mathrm{C}(16)-\mathrm{C}(20)-\mathrm{H}(20 \mathrm{~B})$ & 109.5 \\
$\mathrm{H}(20 \mathrm{~A})-\mathrm{C}(20)-\mathrm{H}(20 \mathrm{~B})$ & 109.5 \\
$\mathrm{C}(16)-\mathrm{C}(20)-\mathrm{H}(20 \mathrm{C})$ & 109.5 \\
$\mathrm{H}(20 \mathrm{~A})-\mathrm{C}(20)-\mathrm{H}(20 \mathrm{C})$ & 109.5 \\
$\mathrm{H}(20 \mathrm{~B})-\mathrm{C}(20)-\mathrm{H}(20 \mathrm{C})$ & 109.5 \\
$\mathrm{C}(1)-\mathrm{N}(1)-\mathrm{C}(5)$ & $124.8(4)$ \\
$\mathrm{C}(1)-\mathrm{N}(1)-\mathrm{C}(8)$ & $117.7(4)$ \\
$\mathrm{C}(5)-\mathrm{N}(1)-\mathrm{C}(8)$ & $117.3(4)$ \\
$\mathrm{C}(9)-\mathrm{O}(3)-\mathrm{C}(14)$ & $115.4(4)$ \\
& \\
\hline
\end{tabular}

Symmetry transformations used to generate equivalent atoms:

$\# 1-x+1 / 3,-y+2 / 3,-z+2 / 3$ 
Table 4. Anisotropic displacement parameters $\left(\AA^{2} \times 10^{3}\right)$ for sqz. The anisotropic displacement factor exponent takes the form: $-2 \pi^{2}\left[h^{2} a^{* 2} U^{11}+\ldots+2 h \mathrm{k} \mathrm{a}^{*} \mathrm{~b}^{*} \mathrm{U}^{12}\right]$

\begin{tabular}{|c|c|c|c|c|c|c|}
\hline & $\mathrm{U}^{11}$ & $\mathrm{U}^{22}$ & $\mathrm{U}^{33}$ & $\mathrm{U}^{23}$ & $\mathrm{U}^{13}$ & $\mathrm{U}^{12}$ \\
\hline$C(1)$ & $23(4)$ & $20(3)$ & $16(4)$ & $-4(3)$ & $-6(3)$ & $12(3)$ \\
\hline$C(2)$ & $22(3)$ & $19(3)$ & $13(3)$ & $-4(3)$ & $1(3)$ & $14(3)$ \\
\hline$C(3)$ & $18(3)$ & $12(3)$ & $20(4)$ & $-2(3)$ & $0(2)$ & $6(2)$ \\
\hline$C(4)$ & $21(3)$ & $15(3)$ & $14(4)$ & $2(3)$ & $3(3)$ & $3(3)$ \\
\hline$C(5)$ & $34(4)$ & $18(3)$ & $24(4)$ & $-7(3)$ & $-10(3)$ & $10(3)$ \\
\hline$C(6)$ & $21(3)$ & $22(3)$ & $29(4)$ & $-4(3)$ & $-5(3)$ & $12(3)$ \\
\hline$C(7)$ & $29(3)$ & $16(3)$ & $26(4)$ & $3(3)$ & $-1(3)$ & $13(3)$ \\
\hline$C(8)$ & $16(3)$ & $20(3)$ & $28(4)$ & $0(3)$ & $3(3)$ & $8(3)$ \\
\hline $\mathrm{C}(9)$ & $23(3)$ & $24(4)$ & $17(4)$ & $0(3)$ & $-2(3)$ & $10(3)$ \\
\hline$C(10)$ & $27(3)$ & $15(3)$ & $29(4)$ & $3(3)$ & $0(3)$ & $5(3)$ \\
\hline$C(11)$ & $28(3)$ & $33(4)$ & $14(4)$ & $9(3)$ & $10(3)$ & $11(3)$ \\
\hline$C(12)$ & $24(3)$ & $20(3)$ & $22(4)$ & $3(3)$ & $2(3)$ & $8(3)$ \\
\hline$C(13)$ & $18(3)$ & $11(3)$ & $24(4)$ & $-2(3)$ & $-5(3)$ & $5(3)$ \\
\hline$C(14)$ & $25(4)$ & $27(4)$ & $34(5)$ & $-3(3)$ & $-4(3)$ & $9(3)$ \\
\hline$C(15)$ & $26(4)$ & $30(4)$ & $39(5)$ & $-5(3)$ & $1(3)$ & $9(3)$ \\
\hline$C(16)$ & $20(3)$ & $21(3)$ & $24(4)$ & $3(3)$ & $6(3)$ & $11(3)$ \\
\hline$C(17)$ & $29(3)$ & $32(4)$ & $33(4)$ & $-8(3)$ & $-10(3)$ & $18(3)$ \\
\hline$C(18)$ & $52(4)$ & $54(5)$ & $31(5)$ & $-8(4)$ & $-16(3)$ & $35(4)$ \\
\hline$C(19)$ & $33(4)$ & $42(4)$ & $31(4)$ & $0(3)$ & $8(3)$ & $20(3)$ \\
\hline$C(20)$ & $26(4)$ & $31(4)$ & $32(4)$ & $-4(3)$ & $-3(3)$ & $13(3)$ \\
\hline $\mathrm{N}(1)$ & $12(2)$ & $16(2)$ & $20(3)$ & $4(2)$ & $3(2)$ & $3(2)$ \\
\hline $\mathrm{O}(1)$ & $18(2)$ & $33(2)$ & $37(3)$ & $4(2)$ & $3(2)$ & $18(2)$ \\
\hline $\mathrm{O}(2)$ & $34(2)$ & $33(2)$ & $31(3)$ & $8(2)$ & $8(2)$ & $20(2)$ \\
\hline $\mathrm{O}(3)$ & $27(2)$ & $16(2)$ & $28(3)$ & $-6(2)$ & $4(2)$ & $3(2)$ \\
\hline $\mathrm{O}(4)$ & $69(3)$ & $36(3)$ & $84(4)$ & $-26(3)$ & $4(3)$ & $11(2)$ \\
\hline $\mathrm{O}(5)$ & $28(2)$ & $55(3)$ & $42(3)$ & $-7(2)$ & $8(2)$ & $10(2)$ \\
\hline
\end{tabular}


Table 5. Hydrogen coordinates $\left(\mathrm{x} 10^{4}\right)$ and isotropic displacement parameters $\left(\AA^{2} \times 10\right.$ 3) for sqz.

\begin{tabular}{|c|c|c|c|c|}
\hline & $\mathrm{x}$ & $\mathrm{y}$ & $\mathrm{z}$ & $\mathrm{U}(\mathrm{eq})$ \\
\hline $\mathrm{H}(6)$ & 691 & 3532 & 3083 & $70(20)$ \\
\hline $\mathrm{H}(7)$ & 1305 & 4030 & 1504 & $16(13)$ \\
\hline $\mathrm{H}(10)$ & -520 & 1180 & 7029 & $44(17)$ \\
\hline $\mathrm{H}(11)$ & -585 & 1503 & 9086 & $31(15)$ \\
\hline $\mathrm{H}(13)$ & 535 & 2809 & 8063 & $19(13)$ \\
\hline $\mathrm{H}(14 \mathrm{~A})$ & -497 & 1043 & 4840 & $34(15)$ \\
\hline $\mathrm{H}(14 \mathrm{~B})$ & -109 & 944 & 5615 & $27(15)$ \\
\hline $\mathrm{H}(17 \mathrm{~A})$ & 182 & 2397 & 12158 & $38(16)$ \\
\hline $\mathrm{H}(17 \mathrm{~B})$ & 52 & 1911 & 11347 & $11(12)$ \\
\hline $\mathrm{H}(18 \mathrm{~A})$ & 917 & 2821 & 10932 & $34(16)$ \\
\hline $\mathrm{H}(18 \mathrm{~B})$ & 787 & 2332 & 10121 & $70(20)$ \\
\hline $\mathrm{H}(18 \mathrm{C})$ & 889 & 2367 & 11730 & $53(18)$ \\
\hline $\mathrm{H}(19 \mathrm{~A})$ & -727 & 1795 & 10858 & $39(16)$ \\
\hline $\mathrm{H}(19 \mathrm{~B})$ & -804 & 2186 & 10023 & $70(20)$ \\
\hline $\mathrm{H}(19 \mathrm{C})$ & -616 & 2295 & 11570 & $46(17)$ \\
\hline $\mathrm{H}(20 \mathrm{~A})$ & -64 & 3008 & 9476 & $48(17)$ \\
\hline $\mathrm{H}(20 \mathrm{~B})$ & 463 & 3115 & 9954 & $36(15)$ \\
\hline $\mathrm{H}(20 \mathrm{C})$ & 64 & 3043 & 11069 & $26(15)$ \\
\hline
\end{tabular}

${ }^{1}$ Connors, K. A. Binding Constants; Wiley: New York, 1987 